\title{
Effect of the choice of WHO International Reference Preparation for thromboplastin on International Normalised Ratios
}

\author{
L Poller, D A Taberner, J M Thomson, J Morris, R S Mibashan, N K Shinton
}

\begin{abstract}
Aims: To compare the International Normalised Ratio (INR) obtained directly with the two types of WHO plain International Reference Preparation for thromboplastin in patients treated with coumarin.

Methods: Prothrombin times were performed in parallel at four centres using WHO human plain IRP (BCT/253) and rabbit plain IRP (RBT/79). Sixty patients and 20 normal controls were tested at each centre. Differences in INR among the centres were assessed by one factor, analysis of variance. The bias for each centre was assessed by the $t$ test.

Results: At all four centres higher INRs were consistently found with the rabbit plain reagent. Two of the centres showed significantly greater bias.

Conclusions: There was a small but significant difference in INR results obtained directly with these two reference reagents at all four centres (mean $7 \cdot 35 \%$ ). This in part may result from the different responsiveness of the two IRP to the coumarin defect or to imprecision of the original ISI calibrations of the two plain WHO IRP. The findings support the adoption of a single master IRP, in accord with WHO recommendations, which would resolve the present anomalous situation.
\end{abstract}

(F Clin Pathol 1993;46:64-66)

The international system for prothrombin time standardisation is based on the calibration of local and commercial thromboplastins in terms of the WHO first primary International Reference Preparation (IRP). ${ }^{1}$ Because supplies of the primary IRP (of human brain origin) were limited, a hierarchical system of thromboplastin calibration using alternative routes of IRP calibration was evolved. Two different plain IRP of human (BCT/253) and rabbit (RBT/79) origin were provided by the WHO for the conventional prothrombin time and a bovine combined (OBT/79), for thromboplastins containing adsorbed plasma. ${ }^{2}$ The WHO replaced its primary IRP of human origin by a second primary WHO human plain IRP $^{3}$ and agreed that future IRP of thromboplastin should be calibrated against the latter. ${ }^{4}$ Additional reference materials have been provided by the International Committee for Standardisation in Haematology ${ }^{5}$ and the $\mathrm{EC}^{26} \mathrm{~A}$ choice of IRP for calibrations is therefore available to commercial manufac- turers and national control laboratories. The provision of IRP plain from rabbit and human sources was to meet the belief that "like to like" species calibration was thought to be more precise, although the alternative of "like to like" responsiveness to the coumarin induced defect of the thromboplastins was an alternative explanation for differences in precision of calibration.

\section{Methods}

The participating centres were as follows: Coventry and Warwickshire General Hospital, Coventry; Royal Hallamshire Hospital, Sheffield; Kings College Hospital, London; and the UK Reference Laboratory for Anticoagulant Reagents and Control, Manchester. The four centres received samples of the human plain WHO IRP (BCT/253) and the rabbit plain WHO IRP (RBT/79) together with a supply of the new rabbit plain reagent to be calibrated. All three reagents were provided in glass sealed ampoules together with reconstitution fluids.

The design of the study was similar to the one undertaken to calibrate the second primary IRP BCT $/ 253$. $^{3}$ Instructions on reconstituting the lyophilised thromboplastins were provided.

Testing was to be performed on five separate days on fresh plasma samples from four normal subjects and 12 patients stabilised for at least six weeks with oral anticoagulants. INR were to be between 1.5 and 4.5 . The exercise was to be completed within four hours of blood being collected. A single exercise was designed as follows: the specimens were collected, by clean venepuncture, into a 1 in 10 volume of sterile trisodium citrate 0.109 to $0.120 \mathrm{~mol} / \mathrm{l}$, into a plastic or polypropylene syringe, and transferred into a non-wettable container. If an evacuated tube was used the brand selected had to be sufficiently siliconised.

First 1-2 hours: collection and preparation of test samples. The blood was centrifuged immediately after withdrawal and the plasma transferred to a non-wettable, stoppered container. Specimens were maintained at room temperature until required for testing.

Second 2 hours: testing of the 16 fresh plasma samples with the three thromboplastins was done according to a randomised sequence provided.

Single determinations were requested and the three prothrombin times on each plasma (one with each thromboplastin) were performed immediately after each other.

The order in which the patients were to be tested was the same as the order of collection. 
The first normal control sample was collected before the treated patients' samples and the fourth normal control collected last, after the patients' samples.

The coagulation end-point was to be based on a manual (tilt tube) technique with a recommended procedure for the Quick test.

A one factor analysis of variance was carried out on the INR biases (rabbit plain-human plain) to investigate differences among the four centres. The size of the bias was assessed for each centre using $t$ tests on the parameters derived from the analysis of variance model.

\section{Results}

Eighty normal subjects and 241 treated patients were tested at the four centres. The results are given in the table below.

The study reveals a highly significant difference between the INR results from the use of the two main WHO IRP for thromboplastin, rabbit plain and human plain (figure). The mean difference at the four centres was $7 \cdot 35 \%$. Higher INR are consistently found with the rabbit plain reagent.

Rabbit plain INR values were significantly higher than human plain values for each centre: Coventry and Warwickshire Hospital $t(59)=2 \cdot 8, \quad \mathrm{p}=0.007 ; \quad$ Kings College Hospital $t(59)=3 \cdot 7, \mathrm{p}=0.0004$; Royal Hallamshire Hospital $t(59)=8 \cdot 0$, $\mathrm{p}<0.0001$; UK Reference Laboratory $t(60)=9.5, \mathrm{p}<0.0001$.

The Royal Hallamshire and the UK Refer-

Effect of the type of WHO IRP on International Normalised Ratios

\begin{tabular}{|c|c|c|c|c|c|}
\hline \multirow[b]{2}{*}{ Centre } & \multirow[b]{2}{*}{$N=$} & \multicolumn{2}{|c|}{ Mean INR values with IRP } & \multirow{2}{*}{$\begin{array}{l}\text { Bias } \\
\text { Rabbit plain- } \\
\text { human plain }\end{array}$} & \multirow{2}{*}{$\begin{array}{l}\text { Bias } \\
95 \% C I\end{array}$} \\
\hline & & Rabbit plain & Human plain & & \\
\hline $\begin{array}{l}\text { Coventry \& Warwickshire } \\
\text { Kings College } \\
\text { Royal Hallamshire } \\
\text { UK Reference Laboratory }\end{array}$ & $\begin{array}{l}60 \\
60 \\
60 \\
61\end{array}$ & $\begin{array}{l}3 \cdot 46 \\
3 \cdot 64 \\
2 \cdot 71 \\
3 \cdot 47\end{array}$ & $\begin{array}{l}3 \cdot 36 \\
3 \cdot 50 \\
2 \cdot 42 \\
3 \cdot 12\end{array}$ & $\begin{array}{l}0 \cdot 10 \\
0 \cdot 14 \\
0 \cdot 29 \\
0 \cdot 35\end{array}$ & $\begin{array}{l}0.03,0.18 \\
0.06,0.21 \\
0.22,0.37 \\
0.28,0.42\end{array}$ \\
\hline
\end{tabular}

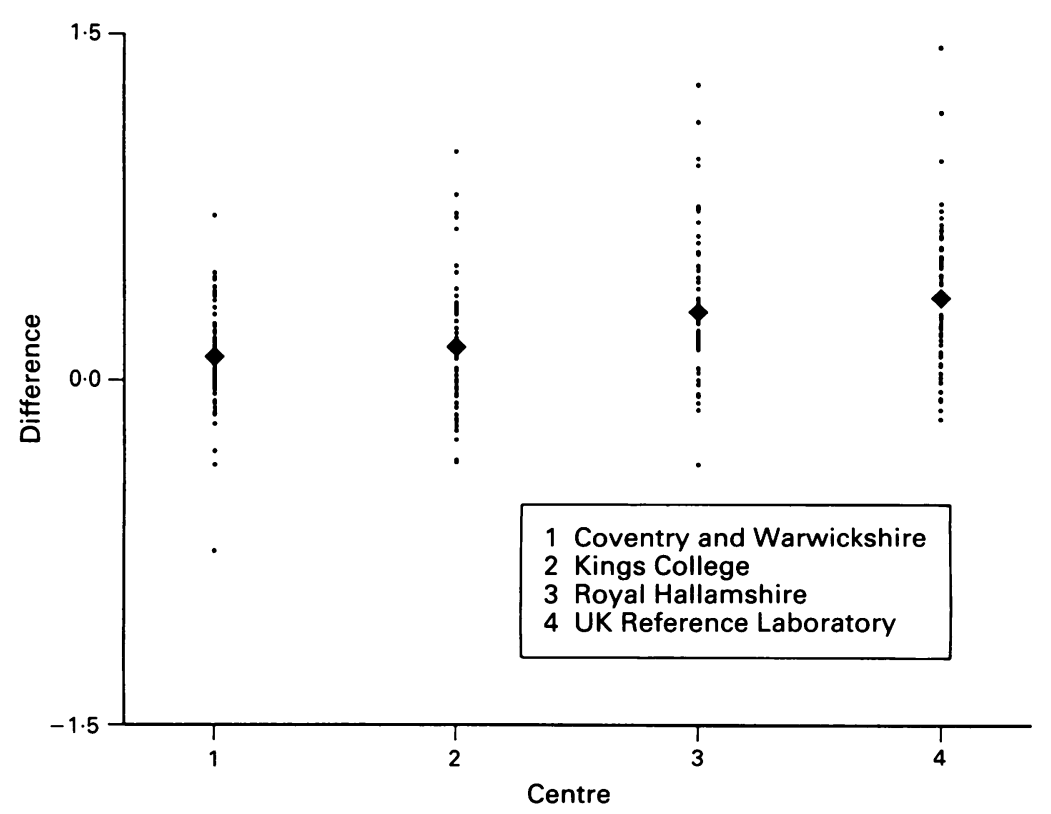

Figure Differences between rabbit plain and human plain INR values. ence Laboratory showed a significantly greater bias compared with Coventry and Warwickshire, and Kings College $(F(3,237)=10 \cdot 6$; $\mathrm{p}<0.001)$.

\section{Discussion}

The present collaborative study shows that significantly higher INR results were obtained with the WHO plain rabbit IRP compared with the human plain. The results show the same trend at each of the four centres to significantly different degrees. A similar pattern of INR results has been consistently found in the series of United Kingdom External Quality Assessment (NEQAS) exercises on lyophilised test samples and in international exercises we have undertaken on behalf of the International Committee for Standardisation in Haematology. There has been difficulty in their interpretation because of the small numbers of samples in the external quality assessment exercises, the variability of the effect with different test samples due to varying responsiveness of individual reagents to depression of different clotting factors, and the uncertainty of the effects of lyophilisation on the test samples. As the differences in the present study were found in fresh plasmas they indicate that the discrepancies cannot be ascribed simply to artefactual changes from lyophilisation in the plasma samples, but can be regarded with confidence as genuine INR differences. The order of differences (mean $7 \cdot 35 \%$ ) is, however, not great in terms of clinical dose of warfarin and of relatively insignificant importance compared with the several fold variation in warfarin dose at different centres before the introduction of the INR system. ${ }^{7}$

The question therefore arises concerning the best route of calibration for a new rabbit plain IRP and for routine commercial rabbit preparations. There are a number of arguments in favour of INR derived from the human IRP calibration route. First, the human plain primary IRP used in this study, as well as being specially designated by WHO, has provided much of the basis for recommendations on therapeutic ranges. This is derived from the cumulative experience in clinical practice and prospective randomised clinical trials with dose control based on human brain thromboplastins calibrated in terms of the human plain IRP. ${ }^{8-10}$ There are also clinical data on therapeutic ranges to support recommendations in terms of the bovine combined preparation (OBT/79) INR which are similar to the human plain. These are derived from the experience largely in the Scandinavian countries and the Netherlands. The implications of the data were incorporated in the above recommendations on therapeutic ranges. The bovine combined type of prothrombin time is, however, a relatively uncommon procedure worldwide and this was one of the reasons put forward for the choice of the second human plain primary IRP as replacement for the first WHO primary IRP which was a combined reagent. ${ }^{11}$

A further reason for the preference for the human plain route of calibration arises from 
the observation that the precision of the INR is partly dependent on the International Sensitivity Index (ISI), the lower the ISI the greater the precision. ${ }^{12}$ The rabbit IRP has a considerably higher ISI $(1 \cdot 41)$ compared with 1.085 for the human plain IRP. Furthermore, the original calibration of the rabbit plain IRP gave a considerably higher SD than did the parallel calibrations of the human plain and the bovine combined, ${ }^{2}$ and was also much higher than the WHO human plain calibration. ${ }^{3}$ This imprecision in ISI calibration is necessarily carried forward with every subsequent ISI calibration based on the rabbit plain IRP.

Finally, the decision to adopt the ISI based on the human plain preparation would accord with the recommendation of the WHO Biological Standards Committee that future IRP should be calibrated against the human plain IRP BCT $/ 253 .^{4}$

1 WHO Expert Committee on Biological Standardisation 33rd Report. Technical Report Series No 687. Geneva:
WHO 1983:25.

2 Hermans J, van den Besselaar AMHP, Loeliger EA, Van der Velde EA. A collaborative study of reference materials for thromboplastins. Thromb Haemostas 1983;50:712-7.

3 Thomson JM, Tomenson JA, Poller L. The calibration of the second international reference preparation for thromboplastin (thromboplastin human plain coded BCT/253) Thromb Haemostas 1984;52:336-42.

4 WHO Expert Committee on Biological Standardisation. 34th Report. Technical Report Series No 700. Geneva: WHO 1984:19.

5 Thomson JM, Darby KV, Poller L. Calibration of BCT/441, the ICSH reference preparation for thromboplastin. Thromb Haemostas 1986;55:379-82.

6 Kirkwood TBL. Calibration of reference thromboplastins and standardisation of the prothrombin time ratio. and standardisation of the proth

7 Poller L, Taberner DA. Dosage and control of oral anticoagulants: an international survey. $\mathrm{Br} \mathcal{F}$ Haemato 1982;51:479-85.

8 ACCP/NHLBI Consensus Conference. Hirsh J, Poller L Deykin $\mathrm{D}$, et al. Optimal therapeutic range for ora anticoagulants. Chest 1989;95:S5-11.

9 British Society for Haematology. Guidelines on oral anticoagulation. 1984. Quoted by Poller L. Laboratory control of anticoagulants. $\mathrm{Br}$ Med $\mathcal{F}$ 1987;294:1184.

10 British Society for Haematology. Guidelines on oral anticoagulation. 2nd edition. $\mathcal{f}$ Clin Pathol 1990;43:177-83.

11 Ingram GIC. The stability of the WHO reference thromboplastin NIBS\&C 67/40. Thromb Haemostas 1979;42: plastin $\mathrm{N}$

12 Taberner DA, Poller L, Thomson JM, Darby KV. Effect of international sensitivity index (ISI) of thromboplastins on precision of international normalised ratios (INR). $\mathcal{f}$ Clin Pathol 1989;42:92-6. 